\title{
Metodologias gamificadas para a educação: uma revisão sistemática
}

\author{
André Luiz de Souza Brito ${ }^{1}$, Charles Andryê Galvão Madeira ${ }^{1}$ \\ ${ }^{1}$ Instituto Metrópole Digital - Universidade Federal do Rio Grande do Norte (UFRN) \\ Natal - Rio Grande do Norte - Brasil \\ andredimd.ufrn.br, charles@imd.ufrn.br
}

\begin{abstract}
Gamification has been a promising approach to tackle student motivation issues in the educational context, through the use of game elements which promote better engagement in the learning process. A lot of research has focused on proposing gamified methodologies for education, but little has been explained about the design and choices made in the construction of the solutions. This work presents a literature review of gamification methodologies with the purpose of observing their creation process, evaluating how and why game elements and tools are usually chosen.
\end{abstract}

Resumo. A gamificação é uma abordagem promissora para ajudar a resolver o problema motivacional dos alunos no contexto educacional, através do uso de elementos de jogos que promovem um maior engajamento no processo de ensino-aprendizagem. Muitos trabalhos de pesquisa têm proposto metodologias gamificadas para a educação. No entanto, pouco tem sido explicado sobre a concepção destas metodologias e sobre como tem sido feita a escolha dos elementos de jogos utilizados na solução para a problemática identificada. Esse trabalho propõe uma revisão sistemática da literatura com intuito de observar como ocorre o processo de concepção dos ambientes educacionais gamificados, desde a escolha dos elementos de jogos até as ferramentas utilizadas.

\section{Introdução}

A desmotivação estudantil é um fenômeno mundial observado em diferentes níveis de escolaridade [Hartnett et al. 2014], podendo ser considerada uma consequência da inadequação das práticas tradicionais de ensino frente aos novos aspectos culturais de gerações mais jovens, principalmente no tocante ao acesso à informação e uso ubíquo das tecnologias como ferramentas do dia-a-dia. Isso demonstra que os jovens alunos necessitam de novos modelos de ensino-aprendizagem que sejam mais interativos, estimulem a proatividade, colaboração, inovação e outras habilidades consideradas essenciais para o novo perfil de formação exigido pela sociedade atual [Coutinho and Lisbôa 2011].

Dentre os esforços empreendidos para ajudar a solucionar este problema, uma área que se destaca é a Gamificação [Deterding et al. 2011], que utiliza elementos e técnicas dos jogos digitais para promover um maior engajamento no processo de ensinoaprendizagem. Muitos trabalhos de pesquisa têm sido direcionados neste sentido, visando propor metodologias gamificadas com aplicação direta em sala de aula ou através de ambientes virtuais de ensino. No entanto, pouco tem sido explicado sobre a concepção destas 
VI Congresso Brasileiro de Informática na Educação (CBIE 2017)

Anais do XXVIII Simpósio Brasileiro de Informática na Educação (SBIE 2017)

metodologias e sobre a escolha dos elementos de jogos utilizados como forma de solução para a problemática identificada. Atualmente, momento em que a gamificação encontra-se no vale da desilusão no Hype Cycle das tecnologias emergentes em educação da Gartner [de Bruyckere 2017], as escolhas dos elementos de jogos utilizados nas soluções gamificadas aparentam ter sido feitas, na grande maioria dos casos, de forma aleatória. Esse modo ad-hoc de escolha dos elementos utilizados pode ter conexão direta com a obtenção de resultados indesejados a médio e longo prazo na maioria das soluções propostas nos últimos anos [Fogel 2015], uma vez que não há garantia que a metodologia contribua de forma eficaz no processo de resolução do problema. Essa também é uma evidência de que o processo de design para a concepção das soluções ainda não é adequado e precisa ser reforçado para garantir a construção de soluções efetivas.

Recentemente, [Ogawa et al. 2016] apresentaram uma revisão sistemática da literatura para identificar como é realizado o processo de avaliação dos ambientes educacionais gamificados. O presente trabalho, diferentemente do de Ogawa, propõe uma revisão sistemática da literatura com intuito de observar como ocorre o processo de concepção dos ambientes educacionais gamificados, o que engloba os elementos de jogos e as ferramentas utilizados. Com esse propósito, o trabalho é estruturado da seguinte forma: a seção 2 apresenta a metodologia de revisão sistemática aplicada, a seção 3 apresenta uma análise sobre os resultados obtidos do processo de revisão e a seção 4 apresenta as conclusões sobre os achados da pesquisa.

\section{Metodologia de Revisão Sistemática}

A metodologia de revisão sistemática executada foi baseada no trabalho de [Petersen et al. 2008] e tem como principal objetivo explorar o estado da arte na área da gamificação na educação, de forma que se tenha um panorama dos principais trabalhos que compõem a vanguarda da área. O processo é composto por diversas etapas: definição das hipóteses de pesquisa, construção do argumento para busca de trabalhos nos principais repositórios científicos e seleção dos trabalhos relevantes, tendo como produto final uma base que representa uma parcela significativa dos esforços em pesquisa na área.

\subsection{Definição das questões de pesquisa}

O primeiro passo foi a definição dos questionamentos que nortearam o processo de análise dos resultados da revisão sistemática. Como o interesse é entender a concepção dos modelos gamificados para trabalhar a motivação estudantil, as seguintes questões foram levantadas:

- Q1: Como é o processo de design da solução? Existe uma investigação prévia do cenário?

- Q2: Que elementos de jogos estão sendo mais usados nos modelos de gamificação para educação?

- Q3: Quais as ferramentas mais utilizadas para dar suporte à gamificação criada?

- Q4: Como a motivação é tratada pela gamificação?

As respostas para esses questionamentos permitirão nos dar uma visão geral do que os pesquisadores pensam antes de conceber uma metodologia gamificada e aplicá-la em um cenário real. 
VI Congresso Brasileiro de Informática na Educação (CBIE 2017)

Anais do XXVIII Simpósio Brasileiro de Informática na Educação (SBIE 2017)

\subsection{Construção do argumento de busca}

Para responder aos questionamentos da seção anterior, foi construído um argumento de busca genérico o suficiente para trazer a maior variedade possível de trabalhos contendo metodologias gamificadas voltadas para a educação:

\section{gamif* and (education or learning) and "student motivation"}

Esse argumento foi utilizado para investigar um conjunto de repositórios científicos, a saber: Scopus, ACM Digital Library, IEEEXplore, Web of Science e SpringerLink. Foi necessário realizar uma adaptação na busca para a base de dados da $A C M$, visto que na época da busca não era possível a utilização dos caracteres de expressão regular simbolizando "coringas" (*, ?), e a expressão de busca foi construída de forma mais explícita (por exemplo, colocando os termos gamification OU gamified OU gamifying, ao invés de gamif*). O resultado dessa busca inicial foi um total de 690 trabalhos, datados a partir do ano de 2011.

A partir desse conjunto inicial não refinado, deu-se início ao processo de seleção dos trabalhos mais relevantes mediante os objetivos do estudo proposto neste trabalho.

\subsection{Seleção de artigos relevantes para a pesquisa}

Para realizar um primeiro filtro do conjunto inicial de trabalhos selecionados, foram adotados os seguintes critérios:

- Critério 1: Eliminação de trabalhos sem disponibilidade do arquivo para leitura completa (download ou online);

- Critério 2: Eliminação de trabalhos duplicados, ou seja, trabalhos que foram encontrados em diferentes bases;

- Critério 3: Eliminação de trabalhos distintos de um mesmo projeto de pesquisa, ou seja, trabalhos diferentes que faziam parte de estudo de longa duração e relatavam momentos ou segmentos diferentes da pesquisa. Nesses casos, deu-se preferência pela versão mais atual e completa do trabalho, também priorizando publicações em periódicos sobre publicação em eventos;

- Critério 4: Eliminação de trabalhos com data de publicação anterior ao ano de 2012, por observa-se que nas bases era a partir desta data que se intensificava as publicações de trabalhos relacionados à gamificação;

- Critério 5: Eliminação de artigos que abordavam a gamificação em áreas não relacionadas à educação;

- Critério 6: Eliminação de artigos que abordavam técnicas de motivação e engajamento distintas da gamificação (game-based learning, por exemplo).

Para a eliminação via critérios 3, 5 e 6 foi efetuada uma leitura dos abstracts e resumos disponíveis para detectar se o artigo atendia às condições aqui pré-definidas. A partir da leitura, foi possível perceber que ainda existe muita confusão com relação à terminologia da área, com um número considerável de trabalhos apresentando propostas de aprendizado baseado em jogos como um modelo de gamificação, o que indica que ainda existe necessidade de conscientização sobre a diferença entre as duas metodologias.

Após esse primeiro filtro, restaram 32 trabalhos, para os quais foi feita uma leitura mais aprofundada da seção de contexto do trabalho e da metodologia aplicada. Nesse 
VI Congresso Brasileiro de Informática na Educação (CBIE 2017)

Anais do XXVIII Simpósio Brasileiro de Informática na Educação (SBIE 2017)

ponto do processo já é possível observar o panorama da pesquisa na área de gamificação nos últimos cinco anos. Observa-se um aumento no número de publicações em eventos e periódicos sobre o tema gamificação, como visto no gráfico da Figura 1, em especial com um aumento significativo de publicações em periódicos nos últimos dois anos.

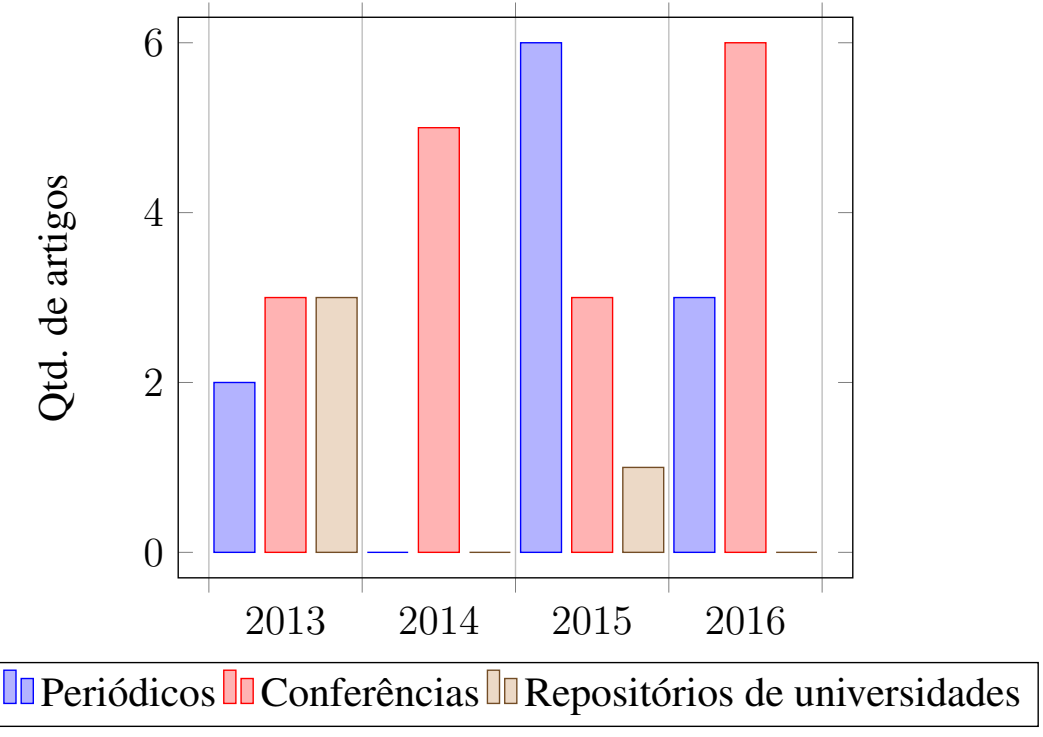

Figura 1. Gráfico contendo a quantidade de artigos analisados que foram publicados a cada ano conforme fontes de publicação.

Outro ponto interessante de observar é com relação aos tipos de estudo executados. Para efeito de classificação, os trabalhos foram agrupados em quatro categorias:

- Revisão da Literatura: trabalhos que traziam uma investigação da literatura concernente ao tema de gamificação;

- Propostas de Modelo Gamificado: trabalhos que apresentavam uma proposta de modelo gamificado, seja um framework conceitual ou uma proposta de ferramenta;

- Estudos de Caso: trabalhos que realizaram experimentação científica através de estudos de caso, seja através do uso de um modelo ou ferramenta própria, ou através do uso de tecnologias já existentes;

- Artigos de Opinião: trabalhos que traziam ponto de vista dos autores sobre o tema da gamificação, mas que não contém um método científico de pesquisa explicitado.

Percebe-se que existe um interesse crescente em pesquisas através de estudos de caso e observação empírica, o que possivelmente é um resultado do amadurecimento do campo de pesquisa que passa a conduzir investigações com um maior rigor científico em termos de técnicas e metodologias, em detrimento dos trabalhos iniciais que detém um caráter mais exploratório, de conhecimento e sensibilização para a importância da área. A Tabela 1 mostra os quantitativos para cada tipo de trabalho considerado.

Dentre os 17 trabalhos que conduziram experimentos de estudo de caso, houve uma forte predileção de realizar a avaliação dos resultados através de métodos quantitativos $(76 \%)$. Uma possível explicação para esse fato pode estar no uso de ferramentas para 
Tabela 1. Quantitativo de trabalhos por tipo de estudo realizado.

\begin{tabular}{|c|c|}
\hline Tipo de Estudo & Total de artigos \\
\hline Artigos de Opinião & 3 \\
Estudos de Caso & 17 \\
Proposta de Modelos Gamificados & 6 \\
Revisão da Literatura & 6 \\
\hline
\end{tabular}

viabilizar os modelos gamificados, que facilitam a coleta de dados a partir da interação dos usuários, permitindo assim que os pesquisadores fizessem comparações com valores previamente obtidos antes de aplicar a gamificação no mesmo cenário. Apesar disso, é interessante reforçar que a coleta de dados qualitativos pode ter um valor muito grande principalmente no entendimento de como os mecanismos de gamificação são percebidos pelos usuários do processo [Iosup and Epema 2014], o que é um feedback valioso para entender como a abordagem afeta o aprendizado e como ela pode ser modificada para engajar um perfil específico de usuário [Barata et al. 2015].

Para refinar ainda mais a base de artigos utilizada, aplicou-se um segundo processo de eliminação, retirando do conjunto de artigos aqueles que não apresentavam uma proposta de metodologia gamificada, mas apenas faziam uma investigação sobre a área. Após esse passo, o conjunto de artigos passou a contar com 21 trabalhos, que foram lidos de forma completa e catalogados para montar o referencial do estado da arte em modelos de gamificação aplicados à educação. A Tabela 2 mostra como o conjunto final foi selecionado a partir das bases de pesquisa, fazendo a ressalva que alguns trabalhos podem ser encontrados em mais de uma delas, mas foram contabilizados apenas em uma das bases (não houve um critério maior de agrupamento nesse sentido, apenas sendo contado para a base onde ele foi inicialmente encontrado).

Tabela 2. Artigos por base de dados (refinado)

\begin{tabular}{|c|c|}
\hline Base de Dados & Total de artigos \\
\hline ACM Digital Library & 6 \\
IEEEXplore & 4 \\
ScienceDirect & 6 \\
Scopus & 4 \\
SpringerLink & 1 \\
\hline Total & 21 \\
\hline
\end{tabular}

Com relação aos níveis de ensino nos quais os modelos gamificados foram aplicados, encontramos uma predominância de aplicações no ensino superior, com $62 \%$ dos trabalhos atuando com alunos desse nível. Uma possível justificativa é que, devido ao trabalho extra que uma metodologia gamificada produz [Iosup and Epema 2014], os pesquisadores realizaram os experimentos em suas próprias turmas, dentro da mesma instituição onde o projeto de pesquisa estava sendo executado, ou em instituições parceiras.

\section{Análise dos Resultados}

Com a base final de 21 artigos, foi realizada uma análise para confrontar os dados com os questionamentos de pesquisa levantados no início do processo de revisão sistemática. A 
VI Congresso Brasileiro de Informática na Educação (CBIE 2017)

Anais do XXVIII Simpósio Brasileiro de Informática na Educação (SBIE 2017)

discussão em cima do que foi observado é feita nas subseções seguintes.

\subsection{Processo de design da solução}

O ponto principal da revisão foi entender o processo de construção das soluções. Dentre os trabalhos encontrados, apenas três apresentam uma investigação, pelo menos parcial, do ambiente em que se deseja aplicar a gamificação, como visto na Figura 2. O entendimento da problemática é um ponto fundamental para que a solução gamificada proposta apresente uma maior probabilidade de funcionar de forma adequada. A partir do momento em que esse passo não é executado no processo de design da solução, não há mais como garantir que a gamificação tenha um efeito positivo. Soluções gamificadas concebidas de forma superficial podem, certas vezes, ter um efeito contrário ao esperado, desmotivando os alunos ao longo do processo ([Attali and Arieli-Attali 2015], [Berkling and Thomas 2013]).

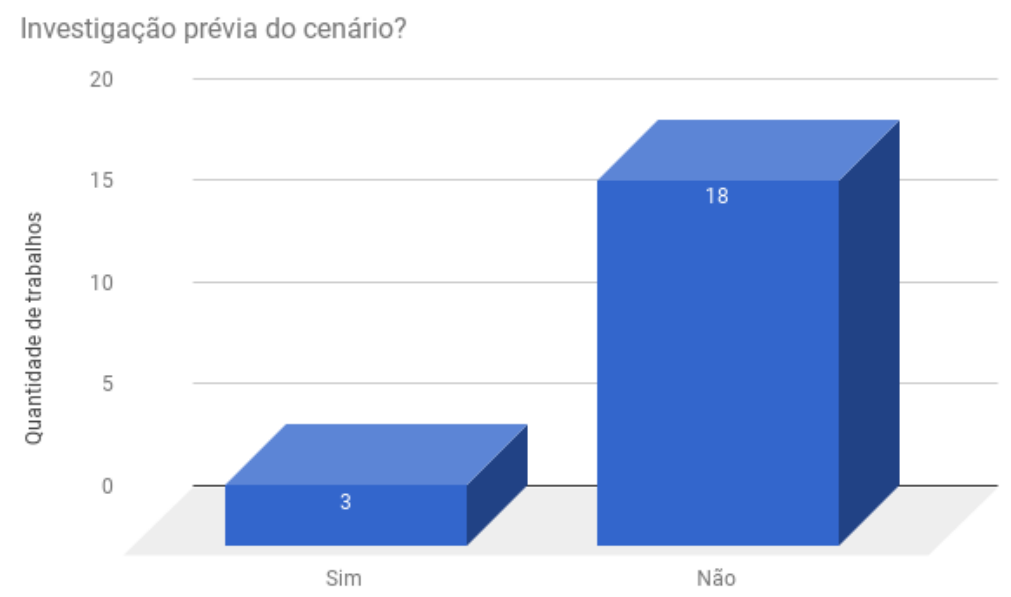

Figura 2. Quantidade de trabalhos que realizam investigação prévia do cenário antes de propor a gamificação.

Dos 18 trabalhos que não realizaram uma investigação do cenário no qual a solução gamificada foi aplicada, 10 não justificam porque escolheram um determinado conjunto de elementos de jogos para montar a solução, ou como esses elementos podem influenciar na motivação dos alunos. É interessante notar que os outros trabalhos escolheram os elementos de jogos baseados na popularidade do seu uso, apenas seguindo aqueles já amplamente utilizados em pesquisas anteriores ([DomíNguez et al. 2013], [Berkling and Thomas 2013]). Esse tipo de conduta não deveria ser adotado pois não garante o sucesso da aplicação da gamificação uma vez que os aspectos motivacionais dos alunos podem ser bem distintos nos diversos cenários investigados pelas pesquisas. Em alguns trabalhos, a gamificação foi projetada com o objetivo de avaliar o efeito de alguns elementos básicos específicos tais quais pontos ([Attali and Arieli-Attali 2015]), medalhas ([Denny 2013], [da Rocha Seixas et al. 2016]) e recompensas ([Hanus and Fox 2015]). Como o foco dessas pesquisas era a investigação da influência de um único elemento, o modelo de gamificação criado acabou sendo restringido pelos objetivos dos trabalhos.

De uma maneira geral, observa-se que a gamificação tem potencial como técnica 
VI Congresso Brasileiro de Informática na Educação (CBIE 2017)

Anais do XXVIII Simpósio Brasileiro de Informática na Educação (SBIE 2017)

para engajar os alunos no ambiente educacional. Porém, o seu processo de concepção deveria ser precedido de uma investigação de como tratar eficazmente os aspectos motivacionais dos alunos, verificando se eles têm interesse neste tipo de solução e estimulando-os para atingir os objetivos de aprendizado. Pelo apurado, poucos trabalhos fazem esse tipo de análise e implantam metodologias gamificadas de uma forma intuitiva e empírica, o que pode ajudar a explicar a existência de sucessos e fracassos em ambientes similares de experimentação, nos permitindo responder ao questionamento Q1.

\subsection{Elementos de jogos utilizados}

A Figura 3 ilustra a frequência de uso de cada um dos elementos de jogos encontrados nas soluções propostas nos artigos selecionados e responde ao questionamento Q2. A análise dos dados comprova que existe um favorecimento no uso de elementos típicos do modelo Points, Badges and Leaderboards - PBL, baseado em elementos mais comumente associados aos jogos (pontos, medalhas e rankings). Esse modelo costuma ser amplamente adotado por sua facilidade de implementação e ampla divulgação, porém estudos apontam que o uso desses recursos de forma superficial não atingem o efeito de motivação desejado [de Marcos et al. 2016]. [Chou 2015] também destaca que apenas o uso ad-hoc de elementos de jogos não garante que uma solução gamificada se torne eficaz. Ao invés disso, ele reforça o ponto de que a gamificação é um processo de design que necessita do entendimento profundo do ambiente e das necessidades do público-alvo.

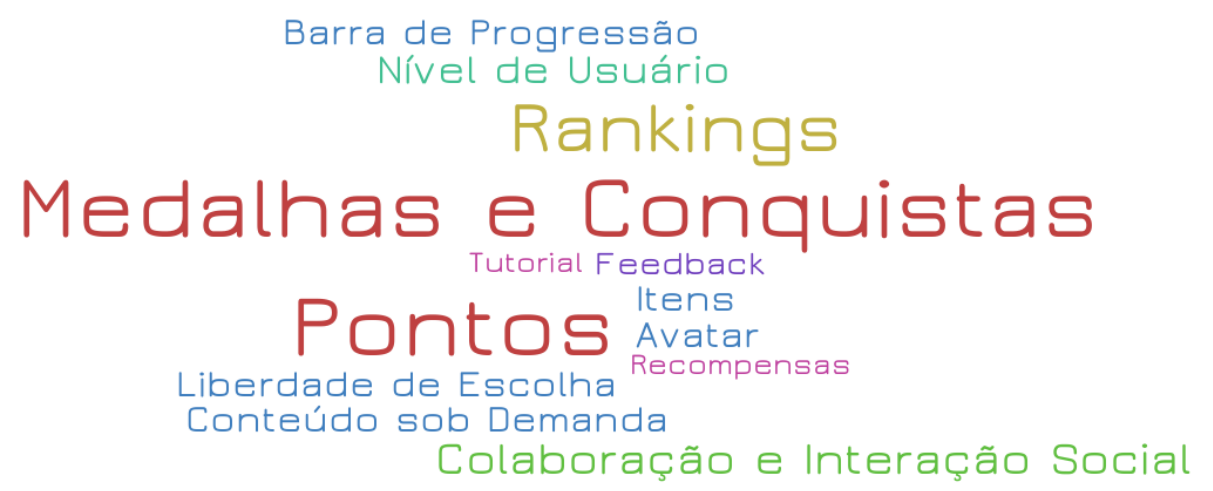

\section{Figura 3. Nuvem de palavras representando a frequência com que cada elemento aparece nas soluções propostas nos artigos.}

Os elementos de jogos podem tratar a motivação a partir de dois aspectos distintos: a motivação extrínseca e a motivação intrínseca [Hanus and Fox 2015]. Motivação extrínseca ocorre quando existe um elemento responsável pela motivação, e seu efeito dura apenas enquanto este elemento está disponível para o aluno, como por exemplo pontos, medalhas e recompensas. Já a motivação intrínseca decorre do prazer inerente de realizar a atividade, sem a necessidade de existir um estímulo externo, e normalmente é associada com a liberdade de escolha, criatividade e expressão. O modelo PBL, e a maior parte dos trabalhos analisados, se apoia fortemente em elementos de motivação extrínseca, o que responde ao questionamento Q4.

Vale a pena destacar que alguns trabalhos iniciaram a exploração de outros elementos de jogos, como o feedback rápido ([Holman et al. 2013]), sistema 
VI Congresso Brasileiro de Informática na Educação (CBIE 2017)

Anais do XXVIII Simpósio Brasileiro de Informática na Educação (SBIE 2017)

de níveis de usuário e progressão no curso ([Berkling and Thomas 2013]), avatares ([Krause et al. 2015]) e missões ([Barata et al. 2015]), e constituem primeiros passos na busca por elementos de jogos que possam tratar a motivação estudantil em um aspecto de médio e longo prazo de forma mais efetiva.

\subsection{Ferramentas utilizadas}

No aspecto de ferramentas utilizadas para experimentação com a gamificação observase como resposta para a questão Q3 uma predileção por ferramentas automatizadas, como sistemas de gestão de conhecimento ou softwares específicos para realização da gamificação. Isso possivelmente acontece como uma forma de mitigar o aumento da carga de trabalho com planejamento de atividades e acompanhamento dos alunos, que passa a ocorrer em um nível mais individualizado.

A maior parte dos trabalhos utiliza ferramentas e sistemas já existentes, como o Moodle ([Gené et al. 2014]), o ClassDojo e ClassBadges ([da Rocha Seixas et al. 2016]), Blackboard ([DomíNguez et al. 2013]) e o Khan Academy ([Ruipérez-Valiente et al. 2016]), dentre outros. Um problema que normalmente se encontra no uso de ferramentas prontas é a falta de flexibilidade, já que a gamificação normalmente fica restrita às possibilidades ofertadas pela ferramenta. Tentando se livrar dessas limitações, alguns trabalhos desenvolveram ferramentas próprias ([Holman et al. 2013], [Dicheva et al. 2014]), ou adotaram um esquema híbrido, executando parte da gamificação em uma ferramenta própria e parte em uma ferramenta ou ambiente já existente na instituição onde o experimento foi executado ([de Marcos et al. 2016]).

É importante refletir sobre o papel da ferramenta na metodologia gamificada. Percebe-se que alguns trabalhos moldaram a gamificação a partir das ferramentas disponíveis, o que pode ser prejudicial à metodologia desenvolvida. Ao invés disso, entendese que primeiro deve ser traçado o plano de gamificação, e depois os ajustes devem ser feitos de acordo com os recursos disponíveis para a implementação. Em alguns casos, é cabível até mesmo uma avaliação de custo benefício entre criar uma nova ferramenta customizada para o modelo idealizado, ou buscar soluções já prontas dentro da comunidade acadêmica e/ou mercado.

\section{Conclusão}

Este trabalho teve como objetivo investigar como o processo de concepção de metodologias gamificadas aplicadas à educação usualmente ocorre. Para isso, foi efetuada uma revisão sistemática da literatura, a partir da qual apurou-se que a maior parte dos trabalhos não realiza uma investigação prévia do cenário no qual a gamificação é aplicada (Q1), escolhendo os elementos de jogos por popularidade de uso ou predileção/intuição dos próprios pesquisadores. Essa falta de um processo apropriado de design da gamificação pode ser um dos fatores que contribuem para a ocorrência de experimentos com resultados inconclusivos ou negativos.

Outro ponto observado é que a maior parte dos trabalhos adotou nas suas soluções elementos de jogos como pontos e medalhas (Q2), que estimulam a motivação extrínseca (Q4) e cujo efeito de engajamento é temporário. Existe uma necessidade de se explorar elementos de jogos que estimulem a motivação intrínseca, com maior capacidade de promover transformações mais duradouras nos estudantes. Alguns desses elementos, como 
VI Congresso Brasileiro de Informática na Educação (CBIE 2017)

Anais do XXVIII Simpósio Brasileiro de Informática na Educação (SBIE 2017)

narrativa e liberdade de escolha do aluno, têm um potencial enorme de aplicação dentro do contexto educacional. Outro ponto de observação foi o conjunto de ferramentas utilizadas, existindo uma predileção pelo uso de ambientes virtuais e ferramentas já existentes para dar suporte à gamificação $(\mathbf{Q 3})$.

Com o panorama obtido pela pesquisa, o próximo passo será na proposição de um processo de construção de modelos gamificados para a educação. Este processo estabelecerá uma sequência de ações que iniciarão com o entendimento da problemática, execução da gamificação no ambiente proposto e análise dos resultados obtidos para melhoria contínua do modelo criado. Espera-se que a definição desse processo contribua significativamente para construção de metodologias gamificadas mais eficientes em resolver os reais problemas de motivação dos alunos.

\section{Referências}

Attali, Y. and Arieli-Attali, M. (2015). Gamification in assessment: Do points affect test performance? Computers \& Education, 83:57-63.

Barata, G., Gama, S., Jorge, J., and Gonçalves, D. (2015). Gamification for smarter learning: tales from the trenches. Smart Learning Environments, 2(1):10.

Berkling, K. and Thomas, C. (2013). Gamification of a software engineering course and a detailed analysis of the factors that lead to it's failure. In Interactive Collaborative Learning (ICL), 2013 International Conference on, pages 525-530. IEEE.

Chou, Y.-k. (2015). Actionable gamification: Beyond points, badges, and leaderboards. Octalysis Media Fremont, CA, USA.

Coutinho, C. P. and Lisbôa, E. S. (2011). Sociedade da informação, do conhecimento e da aprendizagem: desafios para educação no século xxi. Revista de Educação, 18(1):522.

da Rocha Seixas, L., Gomes, A. S., and de Melo Filho, I. J. (2016). Effectiveness of gamification in the engagement of students. Computers in Human Behavior, 58:4863.

de Bruyckere, P. (2017). Gartner's hype cycle for education 2016. https://theeconomyofmeaning.com/2016/08/09/ gartners-hype-cycle-for-education-2016/. Acesso em: $21 \mathrm{de}$ junho de 2017.

de Marcos, L., Garcia-Lopez, E., and Garcia-Cabot, A. (2016). On the effectiveness of game-like and social approaches in learning: Comparing educational gaming, gamification \& social networking. Computers \& Education, 95:99-113.

Denny, P. (2013). The effect of virtual achievements on student engagement. In Proceedings of the SIGCHI conference on human factors in computing systems, pages 763-772. ACM.

Deterding, S., Dixon, D., Khaled, R., and Nacke, L. (2011). From game design elements to gamefulness: defining gamification. In Proceedings of the 15th international academic MindTrek conference: Envisioning future media environments, pages 9-15. ACM. 
VI Congresso Brasileiro de Informática na Educação (CBIE 2017)

Anais do XXVIII Simpósio Brasileiro de Informática na Educação (SBIE 2017)

Dicheva, D., Irwin, K., Dichev, C., and Talasila, S. (2014). A course gamification platform supporting student motivation and engagement. In Web and Open Access to Learning (ICWOAL), 2014 International Conference on, pages 1-4. IEEE.

DomíNguez, A., Saenz-De-Navarrete, J., De-Marcos, L., FernáNdez-Sanz, L., PagéS, C., and MartíNez-HerráIz, J.-J. (2013). Gamifying learning experiences: Practical implications and outcomes. Computers \& Education, 63:380-392.

Fogel, G. (2015). Will $80 \%$ of gamification projects fail? giving credit to gartner's 2012 gamification forecast. <http://www.gameffective.com/ will-80-of-gamification-projects-fail/>. Acesso em: 21 de junho de 2017.

Gené, O. B., Núñez, M. M., and Blanco, Á. F. (2014). Gamification in mooc: challenges, opportunities and proposals for advancing mooc model. In Proceedings of the Second International Conference on Technological Ecosystems for Enhancing Multiculturality, pages 215-220. ACM.

Hanus, M. D. and Fox, J. (2015). Assessing the effects of gamification in the classroom: A longitudinal study on intrinsic motivation, social comparison, satisfaction, effort, and academic performance. Computers \& Education, 80:152-161.

Hartnett, M., St George, A., and Dron, J. (2014). Exploring motivation in an online context: A case study. Contemporary Issues in Technology and Teacher Education, 14(1):31-53.

Holman, C., Aguilar, S., and Fishman, B. (2013). Gradecraft: What can we learn from a game-inspired learning management system? In Proceedings of the Third International Conference on Learning Analytics and Knowledge, pages 260-264. ACM.

Iosup, A. and Epema, D. (2014). An experience report on using gamification in technical higher education. In Proceedings of the 45th ACM technical symposium on Computer science education, pages 27-32. ACM.

Krause, M., Mogalle, M., Pohl, H., and Williams, J. J. (2015). A playful game changer: Fostering student retention in online education with social gamification. In Proceedings of the Second (2015) ACM Conference on Learning@ Scale, pages 95-102. ACM.

Ogawa, A., Klock, A. C. T., and Gasparini, I. (2016). Avaliação da gamificação na área educacional: um mapeamento sistemático. In Brazilian Symposium on Computers in Education (Simpósio Brasileiro de Informática na Educação-SBIE), volume 27, page 440 .

Petersen, K., Feldt, R., Mujtaba, S., and Mattsson, M. (2008). Systematic mapping studies in software engineering. In EASE, volume 8, pages 68-77.

Ruipérez-Valiente, J. A., Muñoz-Merino, P. J., and Kloos, C. D. (2016). An analysis of the use of badges in an educational experiment. In Frontiers in Education Conference (FIE), 2016 IEEE, pages 1-8. IEEE. 